\title{
Análisis estructural y electrónico de los principales confórmeros del complejo $\mathrm{Mg}(\mathrm{II})$ Crisina
}

Juan José MARTÍNEZ MEDINA, María de los Angeles JELLUSSICH \& Nora Beatriz OKULIK

\section{Material Suplementario}

Figura S1. Estructura química del flavonoide crisina.<smiles>O=c1cc(-c2ccccc2)oc2cc(O)cc(O)c12</smiles>

Figura S2. Modelos empleados en la búsqueda conformacional. Los modelos pentacoordinados (acetato monodentado) involucran geometrías piramidales de base cuadrada y bipiramidales de base triangular, mientras que los modelos hexacoordinados (acetato bidentado) involucran geometrías octaédricas. En los dos primeros modelos, A, B y C representan las posiciones alternativas del acetato (monodentado) y las dos moléculas de agua. En el tercer modelo, el acetato bidentado puede coordinar a través de las posiciones $\mathrm{AC}, \mathrm{BC}, \mathrm{AD}, \mathrm{BD}$ o $\mathrm{AB}$, y las dos posiciones restantes son ocupadas por las moléculas de agua.
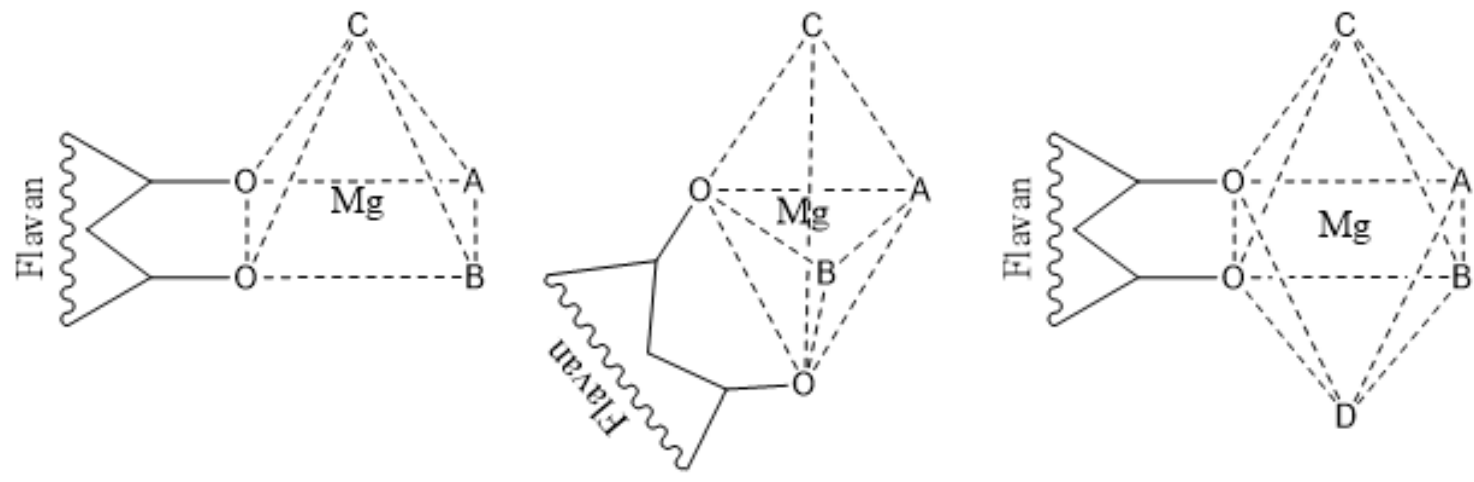

Figura S3. Principales confórmeros de la Familia 1.
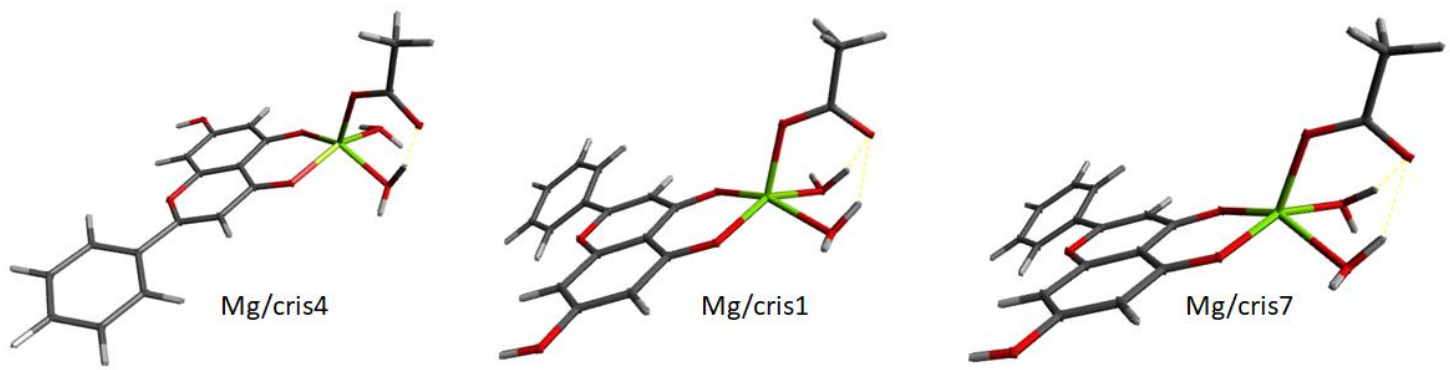
Figura S4. Principales confórmeros de la Familia 2.
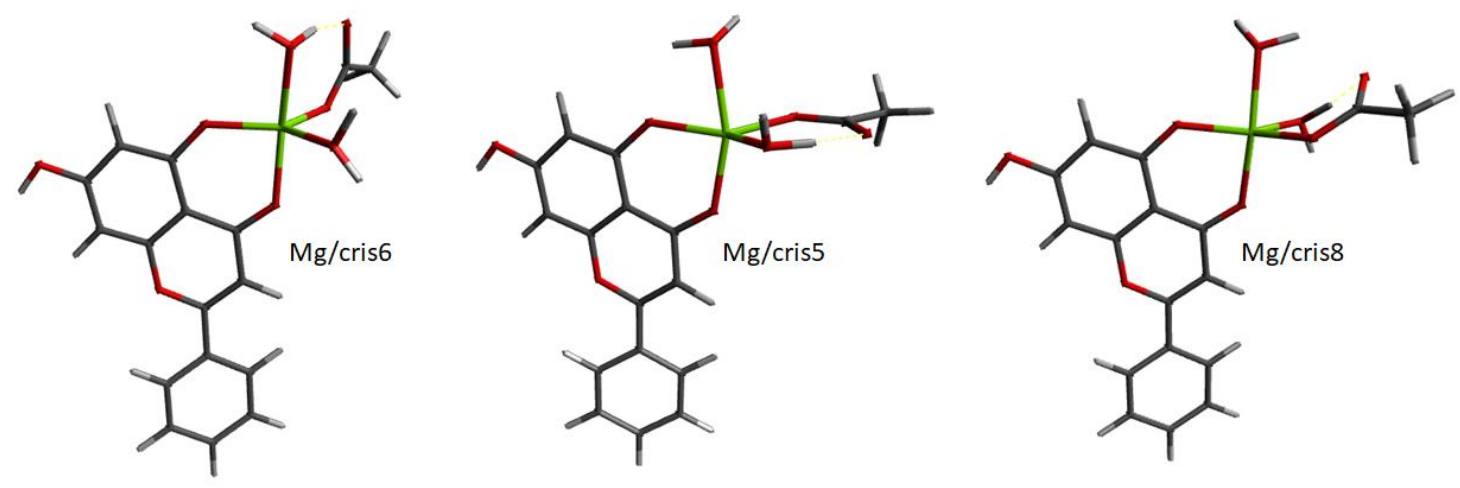

Figura S5. Principales confórmeros de la Familia 3.
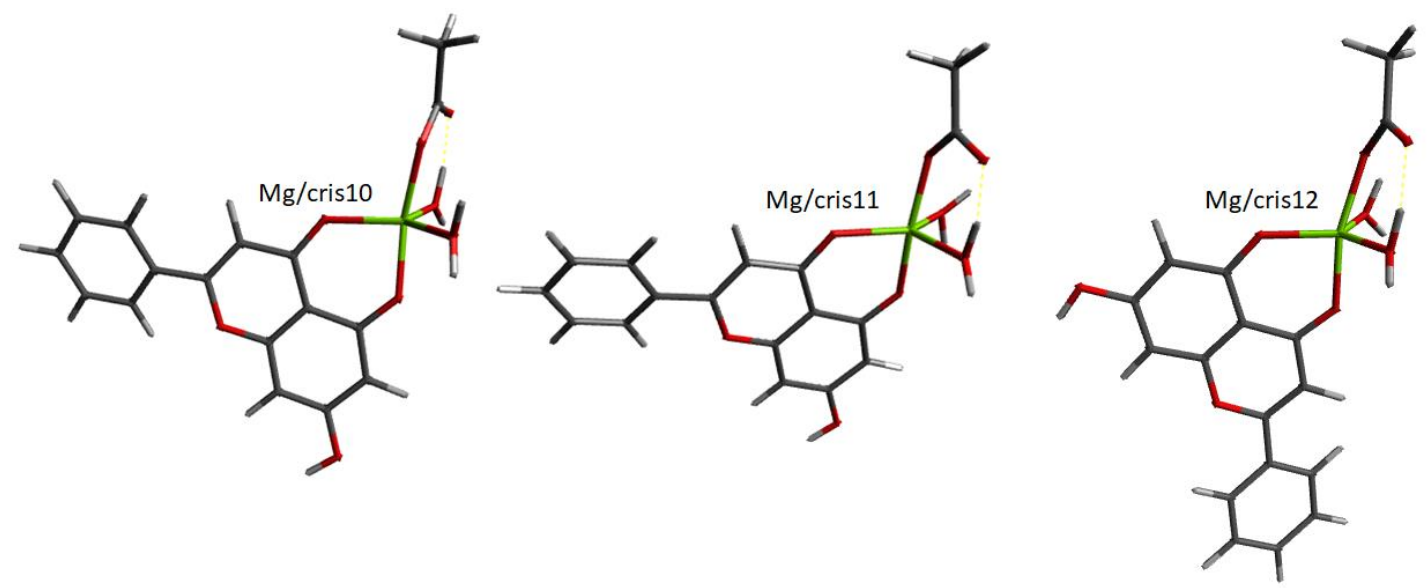

Figura S6. Interacciones débiles reveladas por el método RDG para el confórmero $\mathrm{Mg} /$ cris 10 .
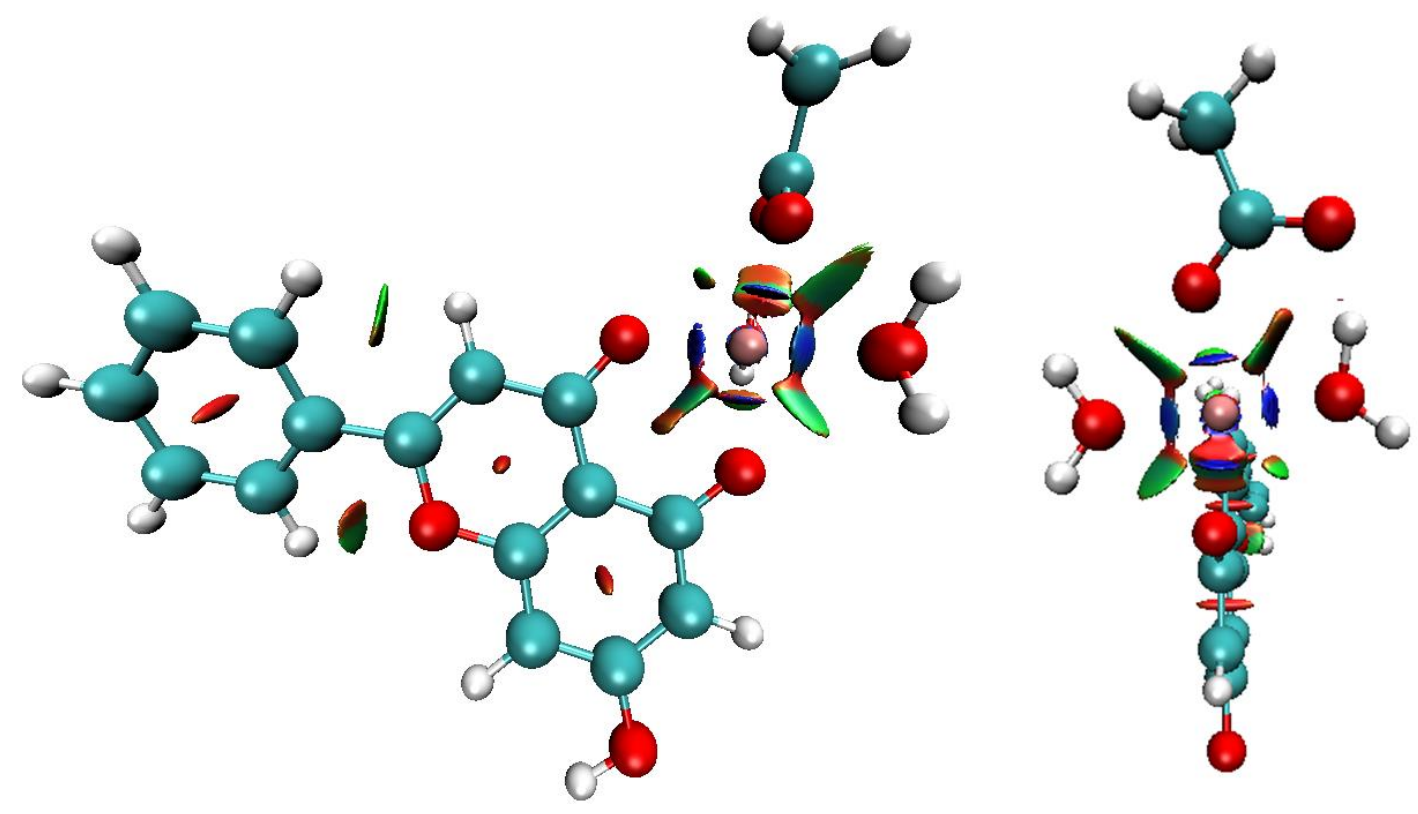
Figura S7. Contribución porcentual de los átomos al orbital HOMO del confórmero $\mathrm{Mg} /$ cris10 calculados a partir de la función de onda extraída de la optimización al nivel B3LYP/6-311++G**.
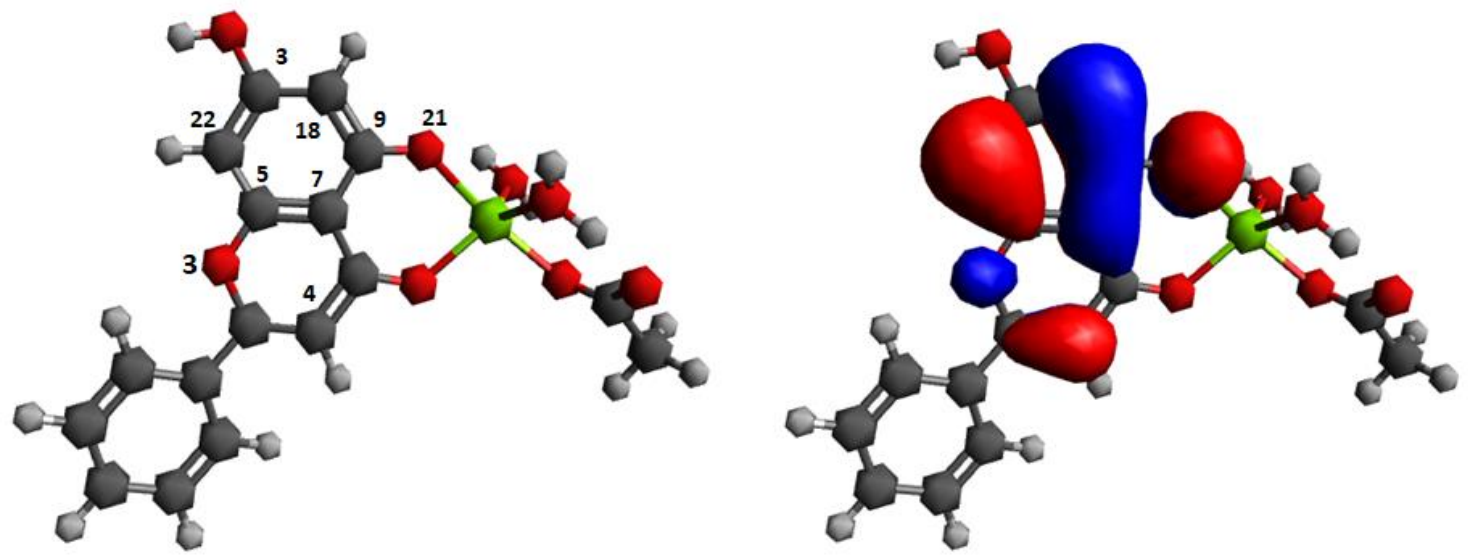

Figura S8. Contribución porcentual de los átomos al orbital LUMO del confórmero $\mathrm{Mg} /$ cris10 calculados a partir de la función de onda extraída de la optimización al nivel B3LYP/6-311++G**.
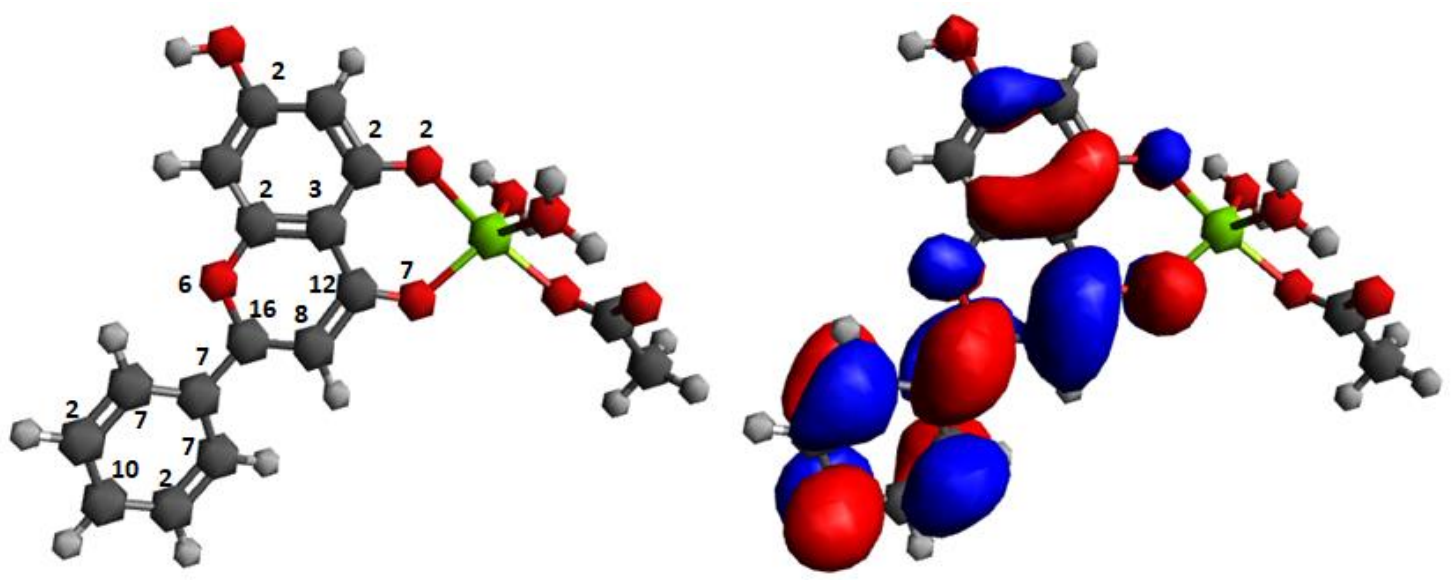

Tabla S1. Principales confórmeros de Mg/cris calculados al nivel B3LYP/6-311++G**. Diferencia energética en relación al confórmero más estable $(\Delta \mathrm{E}, \mathrm{Kcal} / \mathrm{mol})$. Distribución de Maxwell-Boltzmann (\%).

\begin{tabular}{|l|c|c|}
\hline Confórmero & $\boldsymbol{\Delta E}$ & Boltzmann \\
\hline $\mathrm{Mg} /$ cris10 & 0,00 & 9,95 \\
\hline $\mathrm{Mg} /$ cris4 & 0,01 & 9,97 \\
\hline $\mathrm{Mg} /$ cris6 & 0,03 & 11,22 \\
\hline $\mathrm{Mg} /$ cris3 & 0,03 & 10,92 \\
\hline $\mathrm{Mg} /$ cris9 & 0,05 & 12,11 \\
\hline $\mathrm{Mg} /$ cris11 & 0,19 & 12,19 \\
\hline
\end{tabular}


Tabla S2. Principales confórmeros de las Familias 2 y 3 con disminución de la estabilidad (Familia 2 $=\mathrm{Mg} /$ cris6 $>\mathrm{Mg} /$ cris5 $>\mathrm{Mg} /$ cris8 y Familia $3=\mathrm{Mg} /$ cris10 $>\mathrm{Mg} /$ cris11 $>$ $\mathrm{Mg}$ /cris12) y aumento de la distorsión de la bipirámide de base triangular. Los ángulos $\mathrm{O}-\mathrm{Mg}-\mathrm{O}$ de la base trigonal se alejan de $120^{\circ}$ y el ángulo O-Mg-O entre los oxígenos en posición axial se aleja de $180^{\circ}$.

\begin{tabular}{|l|c|c|}
\hline Confórmero & O-Mg-O ecuatorial & O-Mg-O axial \\
\hline $\mathrm{Mg} /$ cris6 & $125-109-126$ & 163 \\
\hline $\mathrm{Mg} / \mathrm{cris} 5$ & $130-95-133$ & 162 \\
\hline $\mathrm{Mg} / \mathrm{cris} 8$ & $138-94-127$ & 171 \\
\hline $\mathrm{Mg} / \mathrm{cris} 10$ & $121-123-116$ & 165 \\
\hline $\mathrm{Mg} / \mathrm{cris} 11$ & $116-116-128$ & 166 \\
\hline $\mathrm{Mg} / \mathrm{cris} 12$ & $118-127-115$ & 161 \\
\hline
\end{tabular}

Tabla S3. Brecha (GAP) entre los orbitales moleculares de frontera HOMO-LUMO.

\begin{tabular}{|l|c|}
\hline Confórmero & GAP (eV) \\
\hline $\mathrm{Mg} /$ cris5 & 3,66 \\
\hline $\mathrm{Mg} /$ cris4 & 3,57 \\
\hline $\mathrm{Mg} /$ cris3 & 3,56 \\
\hline $\mathrm{Mg} /$ cris11 & 3,53 \\
\hline $\mathrm{Mg} /$ cris8 & 3,51 \\
\hline $\mathrm{Mg} /$ cris7 & 3,49 \\
\hline $\mathrm{Mg} /$ cris6 & 3,49 \\
\hline $\mathrm{Mg} /$ cris10 & 3,49 \\
\hline $\mathrm{Mg} /$ cris1 & 3,49 \\
\hline $\mathrm{Mg} /$ cris2 & 3,49 \\
\hline $\mathrm{Mg} /$ cris9 & 3,48 \\
\hline $\mathrm{Mg} /$ cris12 & 3,42 \\
\hline
\end{tabular}

